\title{
Prospective BMI changes in preschool children are associated with parental characteristics and body weight perceptions: the ToyBox-study
}

\author{
Yannis Manios ${ }^{1, *}$, Katrina A Lambert ${ }^{2}$ (1), Eva Karaglani ${ }^{1}$, Christina Mavrogianni ${ }^{1}$, \\ Luis A Moreno Aznar 4,5, Violeta lotova ${ }^{6}$, Anna Świąder-Leśniak7 , Berthold Koletzko8, \\ Greet Cardon ${ }^{9}$, Odysseas Androutsos ${ }^{3}$, George Moschonis ${ }^{10}$, (1) and Toy Box Study \\ Group
}

'Department of Nutrition and Dietetics, School of Health Science and Education, Harokopio University, 70 El Venizelou Avenue, Kallithea 17671, Athens, Greece: ${ }^{2}$ Department of Public Health, School of Psychology and Public Health, La Trobe University, Melbourne, Victoria, Australia: ${ }^{3}$ Department of Nutrition and Dietetics, School of Physical Education, Sport Science and Dietetics, University of Thessaly, Volos, Greece: ${ }^{4}$ GENUD (Growth, Exercise, NUtrition and Development) Research Group, University of Zaragoza, C/Corona de Aragon, Zaragoza, Spain: ${ }^{5}$ School of Health Science (EUCS), University of Zaragoza, C/Domingo Miral s/n, Zaragoza, Spain: ${ }^{6}$ Department of Pediatrics, Medical University Varna, Varna, Bulgaria: ${ }^{7} T h e$ Children's Memorial Health Institute, Warsaw, Poland: ${ }^{8}$ Ludwig-Maximilians-Universität Munich, Dr von Hauner Children's Hospital, University of Munich Medical Centre, Munich, Germany: ${ }^{9}$ Department of Movement and Sports Sciences, Ghent University, Watersportlaan 2, Ghent, Belgium: ${ }^{10}$ Department of Dietetics, Nutrition and Sport, School of Allied Health, Human Services and Sport, La Trobe University, Melbourne, Victoria, Australia

Submitted 15 April 2020: Final revision received 27 January 2021: Accepted 2 March 2021: First published online 12 April 2021

\begin{abstract}
Objective: To examine the effect of the intervention implemented in the ToyBoxstudy on changes observed in age- and sex-specific BMI percentile and investigate the role of perinatal factors, parental perceptions and characteristics on this change.

Design: A multicomponent, kindergarten-based, family-involved intervention with a cluster-randomised design. A standardised protocol was used to measure children's body weight and height. Information was also collected from parents/caregivers via the use of validated questionnaires. Linear mixed effect models with random intercept for country, socio-economic status and school were used.

Setting: Selected preschools within the provinces of Oost-Flanders and WestFlanders (Belgium), Varna (Bulgaria), Bavaria (Germany), Attica (Greece), Mazowieckie (Poland) and Zaragoza (Spain).

Participants: A sample of 6268 preschoolers aged $3.5-5.5$ years ( $51.9 \%$ boys). Results: There was no intervention effect on the change in children's BMI percentile. However, parents' underestimation of their children's actual weight status, parental overweight and mothers' pre-pregnancy overweight/obesity were found to be significantly and independently associated with increases in children's BMI percentile in multivariate modelling.

Conclusions: As part of a wide public health initiative or as part of a counseling intervention programme, it is important to assist parents/caregivers to correctly perceive their own and their children's weight status. Recognition of excessive weight by parents/caregivers can increase their readiness to change and as such facilitate higher adherence to favourable behavioural changes within the family.
\end{abstract} the terms of the Creative Commons Attribution licence (https://creativecommons.org/licenses/by/4.0/), which permits unrestricted re-use, distribution, and reproduction in any medium, provided the original work is properly cited. 
The increased prevalence of childhood obesity and the associated co-morbidities is a major public health concern. Approximately one-third of obese and overweight children continue to follow a higher BMI trajectory throughout the life course ${ }^{(1)}$. It is also well established that adults, who were obese as children, have an increased risk of morbidity and mortality, independent of their current body weight ${ }^{(2,3)}$.

Behaviours which lead to a positive energy balance and consequently to a gradual increase in body weight are established early in life. Consequently, preschool age is a key period for the implementation of behavioural change intervention. Energy balance-related behaviours (EBRB) in preschool years, such as limited engagement into physical activities and increased engagement into sedentary activities (i.e. TV viewing, playing video games etc.), have been associated with obesity in preschool children ${ }^{(4,5)}$. In preschool children, the evidence on relevant associations between obesity and dietary behaviour is also strong but mixed in terms of different dietary behaviours reported to be significantly associated with obesity by different studies ${ }^{(5)}$. However, despite the existing evidence on significant associations between EBRB and childhood obesity in preschool years, the effect of behavioural change interventions that attempt to positively modify EBRB in this young age group is still not adequately explored.

Preschool children do not entirely regulate their own EBRB, as they considerably rely on their parents/guardians. In this context, parental perception of their child's body weight seems to play a significant role on their children's EBRB and weight trajectory. More than half of overweight/obese children have their weight status underestimated by their parents ${ }^{(6)}$. Parents' underestimation of their child's increased body weight could be a significant barrier to the modification of $\mathrm{ERBR}^{(7)}$, since parents who do not recognise their children's overweight status will probably not take any corrective actions. In addition, parental characteristics can have a significant effect on parental modelling, which can subsequently influence children's $\mathrm{EBRB}^{(8)}$. In this regard, parental overweight and obesity produces and sustains an obesogenic environment at home that has been associated with obesity in preschool children in several countries worldwide ${ }^{(9-11)}$ as well as with rapid increases in BMI percentile during preschool years ${ }^{(12)}$.

Further to the effect of social environmental factors on EBRB and weight status of preschool children, a plethora of evidence also highlights the significant effect of factors occurring during early life stages on the subsequent development and growth of children ${ }^{(13,14)}$. There is much to support the linkage of perinatal factors with overweight and obesity in preschool children. As such, maternal pre-pregnancy weight has been associated with a significant risk of childhood overweight and obesity at ages 3, 4 and 5 years ${ }^{(15-20)}$. A systematic review and meta-analysis considering both preschool and elementary age children also reported that pre-pregnancy overweight was associated with two- and threefold increased likelihood of childhood overweight/obesity, respectively ${ }^{(21)}$. Furthermore, gestational weight gain above the recommended threshold has been independently associated with risks of overweight/ obesity in early childhood ${ }^{(22,23)}$, although the effect relative to that of maternal pre-pregnancy overweight has been reported to be smaller ${ }^{(23)}$. In addition, maternal smoking during pregnancy has been associated with a higher BMI in preschool-aged children ${ }^{(24,25)}$, with a systematic review and meta-analysis suggesting an increased risk of overweight in childhood of $50 \%{ }^{(25)}$. Maternal parity has also been associated with a lower risk of childhood overweight ${ }^{(26)}$, although this maybe dependent on breast-feeding ${ }^{(27)}$. Exclusive breast-feeding and later age of introduction of non-breast milk have shown protective effects on preschool overweight/obesity in some ${ }^{(28-30)}$ but not all studies. Despite the well-established relationship between perinatal and early life factors with overweight and obesity at preschool years, their effect on BMI changes in this age group remains to be thoroughly investigated.

The ToyBox-study designed, implemented and evaluated a kindergarten-based, family-involved intervention to 3.5-5.5-year-old children from six European countries, who were followed-up for 1 year. The ToyBox-intervention focused on four different EBRB (water consumption, healthy snacking, sedentary behaviour and physical activity) and has successfully demonstrated decreases in prepacked fruit juice consumption ${ }^{(31)}$ and computer/video games use ${ }^{(32)}$

This study aimed to investigate whether the modification of EBRB attributed to the Toybox intervention could potentially have a positive effect on the changes observed on children's age- and sex-specific BMI percentile. By also recording a wide range of other potentially determining factors, the secondary aim of the present study was to assess the effect of perinatal factors and parental perceptions, socio-demographic and anthropometric characteristics on children's BMI percentile change.

\section{Methods}

\section{ToyBox-study}

The ToyBox-intervention study was a multicomponent, kindergarten-based, family-involved intervention with a cluster-randomised design. The intervention focused on modifying EBRB (see online supplementary material, Supplementary Table 1). A detailed description of the design of the ToyBox-study is provided elsewhere ${ }^{(33)}$.

In brief, a standardised, multistage sampling approach was applied on preschool children aged $3.5-5.5$ years old and their families, who were recruited from randomly selected municipalities within the provinces of OostFlanders and West-Flanders (Belgium), Varna (Bulgaria), Bavaria (Germany), Attica (Greece), Mazowieckie (Poland) 
and Zaragoza (Spain) (see online supplementary material, Supplementary Table 2). Randomisation of the recruited municipalities to intervention and control groups was conducted centrally by the coordinating centre, after the completion of baseline measurements. The municipalities were assigned to the intervention or control group in a 2:1 ratio within each of the three socio-economic status groups. Since the randomisation was conducted at a municipality level, the 309 kindergartens within each municipality were automatically allocated to the intervention or control group. Kindergartens were randomly selected within each municipality.

Intervention mapping protocol was used to plan and develop the standardised ToyBox and provides a systematic, stepwise framework for planning, implementing and evaluating an intervention based on existing scientific literature, theories and research data. Seven hundred kindergarten teachers were trained and delivered the programme in the kindergartens. For evaluation purposes, data were obtained both at baseline and follow-up from more than 5500 parents, indicating significant improvements on the targeted obesogenic behaviours among children and parents, as well as relevant parental practices. The intervention ran for 24 weeks and consisted of four modules on water consumption, healthy snacking, sedentary behaviour and physical activity delivered by the kindergarten teacher. Each model had a four-week 'first focus' and a two-week 'repetition' phase ${ }^{(33)}$.

\section{Data collection}

Data were collected from 7541 preschool children and their parents/caregivers from May to June 2012 and 2013. Children's anthropometrics were measured at baseline and 1-year post intervention, while parents/caregivers self-reported sociodemographic, lifestyle and perinatal information in questionnaires, administered at baseline and the 1-year follow-up.

\section{Energy balance-related behaviour measurement}

Both in the baseline and follow-up measurements, parents/ caregivers were asked to describe the child's usual food and beverage habits over the last 12 months in a FFQ for young children. Items regarding beverage consumption included plain water, soft drinks, light soft drinks, homemade and freshly squeezed fruit juice, pre-packed/bottled fruit juice, tea, smoothies (all kinds), plain milk and sugared or chocolate milk.

Snacking behaviours were also measured using the FFQ completed by the child's guardian, with snacks defined as a small portion of food eaten in between regular meals. Intakes of yogurt (i.e. plain yogurt), sugared or aromatised yogurt, cheese, fresh fruit, raw vegetables, milk-based desserts (e.g. chocolate mousse, ice cream, custard), chocolate and candy bars (e.g. plain chocolate bars, chocolate candy bars), sugar-based desserts (e.g. hard candies, jelly beans, lollipops), cakes, biscuits and salty snacks (e.g. potato chips) were each assessed.

Questions regarding sedentary behaviour were answered by parents/guardians as part of the Primary Caregivers' Questionnaire. The amount of time that children spent on TV/DVD/video viewing, computer/video games use and quiet play on weekdays and weekend days was assessed in three questions of the Primary Caregivers' Questionnaire (one for each SB subcomponent, i.e. TV viewing, computer/video games use and quiet play). For example, 'How much time does your child spend on TV-viewing (a) on weekdays, and (b) on weekend days?'. Possible answer options varied on a nine-point Likert-type scale, ranging from 'never' to 'more than 8 hours/day'.

Physical activity was measured objectively by step counts. Before the start of the intervention, baseline measurements were performed on weekdays from May to June 2012. On those days, researchers visited intervention and control kindergartens and fitted those preschool children with an accelerometer (Belgium) or a pedometer (other countries). One year later, from May to June 2013, follow-up measurements were performed.

\section{Anthropometric measures}

Researchers in all participating centres were rigorously trained to conduct anthropometric measurements following a standardised procedure ${ }^{(34,35)}$. More specifically, researchers conducted two consecutive measurements of children's body weight to the nearest $100 \mathrm{~g}$ and of children's height, to the nearest $1 \mathrm{~mm}$, using the same types of electronic scales (SECA 861 and SECA 813; Seca) and height using portable stadiometers (types SECA 225 and SECA 214; Seca), respectively. If the difference between first and second measurement was $\geq 1 \mathrm{~kg}$ in the case of weight, and $\geq 2 \mathrm{~cm}$ in the case of height, a third measurement was required and the two closest measurements were averaged.

Baseline and follow-up BMI percentile and $z$-scores were calculated for each child using the WHO ANTHRO software for those less than or equal to 60 months, and the WHO ANTHRO-PLUS software for those over 60 months. Children were categorised as: 'underweight', 'normal weight', 'overweight' or 'obese', based on the appropriate $z$-score. BMI percentile change in 1 year was defined as the difference of age- and sex-specific BMI percentile from baseline to 1-year post intervention (BMI percentile post intervention minus baseline BMI percentile) and was considered as the primary response variable in this analysis.

\section{Parental perception on children's body weight}

Parents/caregivers of children were also surveyed concerning their opinion about their child's weight. Cross tabulation between BMI at baseline (per cut-off) and perception of child's weight ('What do you think about your child's weight?'; response on a five-point scale) was used to 
evaluate the guardian's ability to correctly identify their child's body weight status at baseline. Correct perception was considered for: a child grouped as overweight/ obese with parents'/caregivers' response as 'high' or 'very high' child's weight OR a child grouped as normal weight/underweight with response as 'very low' or 'low' or 'normal' child's weight. Misperception was considered for: a child grouped as overweight/obese with response as very low/low/normal child's weight.

\section{Parental socio-demographic and antbropometric characteristics}

Children were categorised as 'native' if both biological parents were born in the country where the questionnaires were administered, whereas all other children as 'nonnative' (i.e. having at least one parent born abroad). Parental body weight and height were self-reported by the parents/caregivers, and their BMI was calculated according to the Quetelet's equation (weight $(\mathrm{kg}) /$ height $^{2}$ $\left(\mathrm{m}^{2}\right)$ ). Parents/caregivers were categorised according to their BMI as 'normal weight' $\left(\leq 24.9 \mathrm{~kg} / \mathrm{m}^{2}\right)$, 'overweight' $\left(\geq 25\right.$ and $\leq 29.9 \mathrm{~kg} / \mathrm{m}^{2}$ ) or 'obese' $\left(\geq 30 \mathrm{~kg} / \mathrm{m}^{2}\right)$. Last, parents/caregivers were asked to report the level of their education.

\section{Perinatal and early life factors}

Mothers were also asked to recall smoking habits during pregnancy (yes/no), pre-pregnancy weight, weight gained during pregnancy based on the classification recommended by the Institute of Medicine ${ }^{(36)}$, multiparous gestation, parity, birth weight and gestational age for the classification into 'small for gestational age' ( $<10$ th percentile), 'appropriate for gestational age' (10th-89th percentile) and 'large for gestational age' ( $\geqslant 90$ th percentile), child's feeding patterns from birth to 6 months of age (exclusive breast-feeding, exclusive formula or mixed), and child's weight and length. Parents/guardians were asked to consult their child's recorded infant's growth chart/medical record and report the weight and length of their child at birth and at the 6th, 7th, 11th and 12th months of their child's life. Using this information, change in weight-for-length $Z$-score from birth to 6 months of age was calculated and then classified into 'poor' $(<-1 Z$ score), 'average' ( -1 to $+1 Z$-score) and 'rapid' $(>+1 Z$ score) weight gain during infancy.

\section{Statistical analysis}

Differences between the control and intervention groups in descriptive variables were calculated by the $\chi^{2}$ test. To account for the nested and hierarchical nature of the sampling linear mixed effect models were used to assess associations between BMI percentile change in 1 year and (i) intervention and (ii) perinatal factors, parental sociodemographic and anthropometric characteristics. Random intercepts were used for country, socio-economic status and kindergarten, with fixed mean for each country and the intercept varying among socio-economic status and kindergarten within socio-economic status.

A multivariate model was produced, containing the intervention effect and all factors found in univariate analysis to be significantly $(P<0.05)$ associated with change in BMI percentile, which were then eliminated from the multivariate model if not significant. Factors with potential collinearity issues were assessed separately and the strongest variable retain in the final model. We also tested for an interaction between the intervention and those factors that were found to be significantly $(P<0.05)$ associated with BMI percentile changes, in order to assess whether these factors could influence the effect of the intervention.

Regression coefficients are presented as beta with $95 \%$ CI. Statistical analysis was performed in R statistical programme (version 3.5.3 (2019-03-11)). The level of significance was set as $P<0.05$.

\section{Results}

Table 1 presents the descriptive characteristics of the study sample. In total, 6268 children $(51.9 \%$ boys, mean age at baseline $4.74 \pm 0.44$ years) provided valid height and body weight data at baseline and follow-up. Previous attrition analyses $^{(32)}$ showed no significant differences in age, sex and group of preschoolers who were lost to follow-up and those who were included (95\% Clage $=0.93,1.16$; $95 \%$ CIsex $=0 \cdot 92,1 \cdot 14 ; 95 \%$ CIgroup $=0 \cdot 91,1 \cdot 52)$. At baseline, the majority of children were under/normal weight ( $89.4 \%$ ), with $7.8 \%$ overweight and $2.8 \%$ obese. The mean change in BMI percentile was -1.48 (SD 13.49), with 907 children (14.5\%) increasing their BMI percentile by more than $10 \%$. The weight status of overweight/obese children was underestimated by $70 \cdot 6 \%$ of their parents or primary guardians (470/666 overweight/obese children; $7.5 \%$ of total sample), and parents/guardians were more likely to underestimate the body weight of male children $(P<0.001)$. Conversely, only $2.3 \%$ of parents/guardians overestimated the weight of their child. Just below $70 \%$ of families had at least one parent/guardian who was overweight/obese.

Table 2 summarises the findings derived from the univariate models testing the effect of the intervention, perinatal factors, parental socio-demographic and anthropometric characteristics on the changes observed in children's BMI percentile. The intervention had no significant effect on the change in BMI percentile. Regarding the effect of parental characteristics, parents'guardians' underestimation of children's weight status at baseline was associated with an increase in children's BMI percentile (1.41, 95\% CI 0.10, 2.72 ). Furthermore, children with only one or both parents being overweight/obese had a 2.47 (95\% CI 1.66, 3.29) and a 4.00 (95\% CI 2.94, 5.06) increase in BMI percentile, respectively, compared with those children whose parents were under/normal weight. As far as the effect of perinatal factors was concerned, overweight/obesity in mothers before 
Table 1 Socio-demographic, parental characteristics and perinatal factors in preschool children from six European countries $(n$ 6268)

\begin{tabular}{|c|c|c|c|c|c|c|c|}
\hline & \multicolumn{2}{|c|}{$\begin{array}{c}\text { Total sample } \\
(n \text { 6268) }\end{array}$} & \multicolumn{2}{|c|}{$\begin{array}{l}\text { Control } \\
(n 2271)\end{array}$} & \multicolumn{2}{|c|}{$\begin{array}{l}\text { Intervention } \\
\text { (n 3997) }\end{array}$} & \multirow[b]{2}{*}{$P$-value } \\
\hline & $n$ & $\%$ & $n$ & $\%$ & $n$ & $\%$ & \\
\hline Gender & & & & & & & 0.982 \\
\hline Female & 3011 & $48 \cdot 0$ & 1090 & $48 \cdot 0$ & 1921 & $48 \cdot 1$ & \\
\hline Male & 3257 & $52 \cdot 0$ & 1181 & $52 \cdot 0$ & 2076 & $51 \cdot 9$ & \\
\hline European country & & & & & & & $<0.001$ \\
\hline Belgium & 1203 & $19 \cdot 2$ & 498 & $21 \cdot 7$ & 705 & $17 \cdot 6$ & \\
\hline Bulgaria & 879 & $14 \cdot 0$ & 256 & $11 \cdot 3$ & 623 & $15 \cdot 6$ & \\
\hline Germany & 988 & $15 \cdot 8$ & 384 & $16 \cdot 9$ & 604 & $15 \cdot 1$ & \\
\hline Greece & 1012 & $16 \cdot 1$ & 285 & 12.5 & 727 & $18 \cdot 2$ & \\
\hline Poland & 1320 & $21 \cdot 1$ & 536 & 23.6 & 784 & $19 \cdot 6$ & \\
\hline Spain & 866 & $13 \cdot 8$ & 312 & 13.7 & 554 & $13 \cdot 9$ & \\
\hline Parents'/guardian' perception of child's weight status & & & & & & & 0.856 \\
\hline Correct/over & 5797 & 92.5 & 2103 & $92 \cdot 6$ & 3694 & $92 \cdot 4$ & \\
\hline Underestimation & 470 & 7.5 & 168 & $7 \cdot 4$ & 302 & $7 \cdot 6$ & \\
\hline Children's weight status at baseline & & & & & & & 0.227 \\
\hline Underweight/normal & 5601 & $89 \cdot 4$ & 2038 & 89.7 & 3563 & $89 \cdot 2$ & \\
\hline Overweight & 490 & 7.8 & 180 & 7.9 & 310 & $7 \cdot 8$ & \\
\hline Obese & 176 & $2 \cdot 8$ & 53 & $2 \cdot 3$ & 123 & $3 \cdot 1$ & \\
\hline Nationality & & & & & & & 0.076 \\
\hline Native & 5617 & $90 \cdot 0$ & 2056 & 91.7 & 3561 & $90 \cdot 3$ & \\
\hline Non-native parent/s & 568 & $9 \cdot 6$ & 186 & $8 \cdot 3$ & 382 & $9 \cdot 7$ & \\
\hline Parental weight status & & & & & & & 0.593 \\
\hline No parent overweight/obese & 1533 & $30 \cdot 1$ & 578 & $31 \cdot 0$ & 955 & $29 \cdot 6$ & \\
\hline One parent overweight/obese & 2616 & 51.4 & 945 & $50 \cdot 6$ & 1671 & 51.9 & \\
\hline Both parents overweight/obese & 938 & 18.4 & 343 & $18 \cdot 4$ & 595 & $18 \cdot 5$ & \\
\hline Smoking during pregnancy & & & & & & & 0.814 \\
\hline No smoking & 4721 & 78.2 & 1723 & 78.4 & 2998 & $78 \cdot 1$ & \\
\hline Smoking & 1316 & $21 \cdot 8$ & 475 & 21.6 & 841 & 21.9 & \\
\hline Gestational weight gain ${ }^{* *}$ & & & & & & & 0.265 \\
\hline Meet IOM recommended weight gain & 3507 & $62 \cdot 7$ & 1259 & 61.7 & 2248 & $63 \cdot 3$ & \\
\hline Exceed IOM recommended weight gain & 2087 & $37 \cdot 3$ & 781 & $38 \cdot 3$ & 1306 & $36 \cdot 7$ & \\
\hline Gestational age & & & & & & & 0.025 \\
\hline Full-term ( $\geq 37$ week $)$ & 5720 & $91 \cdot 3$ & 2097 & $92 \cdot 3$ & 3623 & $90 \cdot 6$ & \\
\hline Pre-term (<37 weeks) & 548 & 8.7 & 174 & 7.7 & 374 & 9.4 & \\
\hline Multiple birth & & & & & & & 0.035 \\
\hline Single gestation & 5606 & 93.3 & 2073 & 94.2 & 3533 & $92 \cdot 7$ & \\
\hline Multiple gestation & 405 & $6 \cdot 7$ & 128 & $5 \cdot 8$ & 277 & $7 \cdot 3$ & \\
\hline Birth weight & & & & & & & 0.061 \\
\hline Small for gestational age & 598 & $10 \cdot 4$ & 192 & $9 \cdot 2$ & 406 & $11 \cdot 1$ & \\
\hline Normal for gestational age & 4618 & $80 \cdot 2$ & 1709 & 81.5 & 2909 & $79 \cdot 4$ & \\
\hline Large for gestational age & 545 & 9.5 & 195 & $9 \cdot 3$ & 350 & 9.5 & \\
\hline Feeding patterns during infancy & & & & & & & 0.053 \\
\hline Formula & 717 & $11 \cdot 8$ & 258 & 11.7 & 459 & $11 \cdot 8$ & \\
\hline Mixed & 4359 & 71.7 & 1547 & $70 \cdot 2$ & 2812 & 72.5 & \\
\hline Breastfeed only & 1006 & $16 \cdot 5$ & 398 & $18 \cdot 1$ & 608 & $15 \cdot 7$ & \\
\hline Growth velocity & & & & & & & 0.232 \\
\hline Normal growth velocity & 2998 & 61.6 & 1111 & $62 \cdot 7$ & 1887 & $61 \cdot 0$ & \\
\hline Retarded growth velocity & 962 & $19 \cdot 8$ & 353 & $19 \cdot 9$ & 609 & $19 \cdot 7$ & \\
\hline Rapid growth velocity & 904 & $18 \cdot 6$ & 307 & $17 \cdot 3$ & 597 & $19 \cdot 3$ & \\
\hline
\end{tabular}

${ }^{*} P$-value derived from $\chi^{2}$ test between control and intervention.

${ }^{\star \star}$ Gestational weight gain recommendations provided by the Institute of Medicine (IOM).

pregnancy was associated with an increase $(2 \cdot 32,95 \% \mathrm{CI}$ $1.41,3.23)$ in children's BMI percentile, while mother's age at pregnancy was associated with a decrease (-0.10, $95 \% \mathrm{CI}-0 \cdot 20,-0 \cdot 01)$ in BMI percentile, but only in girls. In addition, children whose mothers exceeded the recommended weight gain during pregnancy and had a higher gestational age significantly increased their BMI percentiles by 1.15 (95\% CI $0.43,1.86)$ and 0.25 (95\% CI $0.07,0.43)$, respectively. Mixed feeding during infancy was also found to be significantly associated with an increase in BMI percentile (1.86, $95 \%$ CI $0 \cdot 33,3 \cdot 40)$ compared with formula feeding, but this was observed only in boys. Girls whose mothers smoked during pregnancy significantly increased their BMI percentiles by 1.42 (95\% CI 0.28, 2.56), compared with girls whose mothers did not smoke at pregnancy. Decreases in BMI percentile of -1.26 (95\% CI $-2.63,-0.11)$ and -1.48 (95\% CI -2.59, -0.38) were also observed for those children who were born first and small for gestational age, compared with those born at a rank other than first and appropriate for gestational age, respectively.

The findings derived from the multivariate model that examined the intervention effect and all factors found in 
Table 2 Effect of parental characteristics and perinatal factors on the change in the age-and sex-specific BMI percentile over the 1-year period of the ToyBox intervention - univariate modelling ${ }^{*}$

\begin{tabular}{|c|c|c|c|c|c|c|}
\hline \multirow[b]{2}{*}{ Factor } & \multicolumn{2}{|c|}{ Total sample } & \multicolumn{2}{|c|}{ Boys } & \multicolumn{2}{|c|}{ Girls } \\
\hline & Estimate & $95 \% \mathrm{Cl}$ & Estimate & $95 \% \mathrm{Cl}$ & Estimate & $95 \% \mathrm{Cl}$ \\
\hline \multicolumn{7}{|l|}{ Treatment arm } \\
\hline Control & Reference & & Reference & & Reference & \\
\hline Intervention & 0.47 & $-0.37,1.32$ & 0.97 & $-0 \cdot 24,2 \cdot 18$ & 0.78 & $-0.26,1.83$ \\
\hline \multicolumn{7}{|c|}{ Parents'/guardian' perception of child's weight status } \\
\hline Correct estimation & Reference & & Reference & & Reference & \\
\hline Underestimation & 1.41 & $0 \cdot 10,2 \cdot 72$ & $2 \cdot 81$ & $0.97,4.64$ & 1.44 & $-0.48,3.37$ \\
\hline \multicolumn{7}{|l|}{ Parent's Nationality } \\
\hline Native & Reference & & Reference & & Reference & \\
\hline Non-native parent(s) $v$. Native & 0.14 & $-1 \cdot 01,1 \cdot 30$ & -0.67 & $-2 \cdot 34,0.95$ & -0.62 & $-2 \cdot 20,0.95$ \\
\hline \multicolumn{7}{|l|}{ Parental weight status } \\
\hline No parent overweight/Obese & Reference & & Reference & & Reference & \\
\hline 1 parent overweight/obese & 2.47 & $1.66,3.29$ & 2.98 & $1 \cdot 78,4 \cdot 18$ & 2.62 & $1.49,3.75$ \\
\hline Both parents overweight/obese & 4.00 & $2.94,5.06$ & 3.91 & $2.36,5.47$ & 4.40 & $2.93,5.87$ \\
\hline \multicolumn{7}{|l|}{ Parental education } \\
\hline Mother's education ( $<7$ years) & Reference & & Reference & & Reference & \\
\hline $7-12$ years & -0.74 & $-3.97,2.49$ & 0.55 & $-4 \cdot 70,5 \cdot 80$ & -0.61 & $-4.73,3.52$ \\
\hline $13-14$ years & -0.98 & $-4 \cdot 21,2 \cdot 25$ & -0.16 & $-5.41,5.08$ & -0.92 & $-5.03,3.18$ \\
\hline $15-16$ years & $-1 \cdot 18$ & $-4.41,2.04$ & -0.24 & $-5.47,4.99$ & $-1 \cdot 16$ & $-5 \cdot 27,2.94$ \\
\hline More than 16 years & -0.76 & $-3.97,2.44$ & 1.01 & $-4 \cdot 20,6 \cdot 21$ & -0.72 & $-4 \cdot 78,3 \cdot 35$ \\
\hline Father's education ( $<7$ years) & Reference & & Reference & & Reference & \\
\hline $7-12$ years & -1.80 & $-4.54,0.93$ & -2.99 & $-7 \cdot 22,1.23$ & -0.32 & $-3.94,3.30$ \\
\hline $13-14$ years & -1.47 & $-4.21,1.27$ & -2.25 & $-6.49,1.99$ & -0.75 & $-4 \cdot 38,2 \cdot 87$ \\
\hline $15-16$ years & -1.35 & $-4 \cdot 10,1 \cdot 40$ & -2.79 & $-7.05,1.46$ & -0.26 & $-3.90,3.38$ \\
\hline More than 16 years & -0.74 & $-3.47,1.98$ & $-1 \cdot 16$ & $-5 \cdot 37,3.05$ & -0.04 & $-3.64,3.56$ \\
\hline \multicolumn{7}{|l|}{ Maternal pre-pregnancy weight status } \\
\hline Under/normal weight & Reference & & Reference & & Reference & \\
\hline Overweight/Obesity & 2.32 & $1.41,3.23$ & $2 \cdot 28$ & $0.95,3.62$ & 2.36 & $1.14,3.59$ \\
\hline Maternal age at pregnancy (years) & -0.08 & $-0.15,0.00$ & 0.05 & $-0.05,0.16$ & $-0 \cdot 10$ & $-0.20,-0.01$ \\
\hline \multicolumn{7}{|l|}{ Smoking during pregnancy } \\
\hline Not smoking & Reference & & Reference & & Reference & \\
\hline Smoking & 0.20 & $-0.80,1 \cdot 20$ & $2 \cdot 10$ & $-0.83,3.36$ & 1.42 & $0.28,2.56$ \\
\hline \multicolumn{7}{|l|}{ Gestational weight gain } \\
\hline Meet IOM recommended weight gain & Reference & & Reference & & Reference & \\
\hline Exceed IOM recommended weight gain & 1.15 & $0.43,1.86$ & 1.05 & $0.01,2.09$ & 0.82 & $-0 \cdot 16,1 \cdot 80$ \\
\hline Gestational age (weeks) & 0.25 & $0.07,0.43$ & 0.22 & $-0.05,0.49$ & 0.15 & $-0.10,0.39$ \\
\hline \multicolumn{7}{|l|}{ Parity } \\
\hline Other & Reference & & Reference & & Reference & \\
\hline First born & -1.26 & $-2 \cdot 63,-0 \cdot 11$ & -0.60 & $-2 \cdot 62,1.41$ & -0.29 & $-2.13,1.55$ \\
\hline \multicolumn{7}{|l|}{ Birth weight } \\
\hline AGA/LGA & Reference & & Reference & & Reference & \\
\hline $\begin{array}{l}\text { SGA } \\
\text { AGA/SGA }\end{array}$ & $\begin{array}{c}-1.48 \\
\text { Reference }\end{array}$ & $-2 \cdot 59,-.38$ & $\begin{array}{c}-0.86 \\
\text { Reference }\end{array}$ & $-2 \cdot 50,0 \cdot 78$ & $\begin{array}{c}-1.66 \\
\text { Reference }\end{array}$ & $-3 \cdot 16,-0 \cdot 16$ \\
\hline LGA & 1.14 & $-0.01,2.29$ & 0.73 & $-1 \cdot 05,2 \cdot 50$ & 0.90 & $-0.62,2.41$ \\
\hline \multicolumn{7}{|l|}{ Breast-feeding } \\
\hline Formula & Reference & & Reference & & Reference & \\
\hline Mixed & 0.42 & $-0.67,1.51$ & 1.86 & $0.33,3.40$ & 1.31 & $-0.16,2.75$ \\
\hline Breastfeed only & -0.10 & $-1.43,1.23$ & 0.63 & $-1 \cdot 23,2.50$ & 0.97 & $-0.78,2.72$ \\
\hline \multicolumn{7}{|l|}{ Growth velocity in the first 6 months } \\
\hline Normal growth velocity & Reference & & Reference & & Reference & \\
\hline Retarded growth velocity & 0.52 & $-0.42,1.47$ & 0.44 & $-0.93,1.81$ & -0.31 & $-1.63,1.01$ \\
\hline Rapid growth velocity & -0.12 & $-1.09,0.85$ & 0.53 & $-0.92,1.98$ & -0.28 & $-1.59,1.03$ \\
\hline
\end{tabular}

IOM recommendations = recommendations by the Institute of Medicine (IOM) 2009 report; AGA, normal for gestational age; LGA, large for gestational age; SGA, small for gestational age.

${ }^{\star}$ Estimates from linear mixed effect models with random intercept for country, socio-economic status and school, and fixed effect adjustment for BMI pre-intervention.

$0 \cdot 22,3 \cdot 21)$. Similarly, children with one $(2 \cdot 34,95 \% \mathrm{CI}$ $1 \cdot 50,3 \cdot 18)$ or both parents $(2 \cdot 87,95 \%$ CI $1 \cdot 61,4 \cdot 13)$ being overweight/obese, as well as children whose mother was overweight/obese before pregnancy $(2 \cdot 03,95 \%$ CI $0 \cdot 89$, $3 \cdot 17$ ), were also found to significantly increase their BMI percentile, compared with children with under/normalweight parents. Girls born small for gestational age showed associated winc ince in BMI percentile $(1.72,95 \%$

the univariate analyses to be significantly associated with the change in BMI percentile are displayed in Table 3. The non-significant effect of the intervention observed at the univariate analysis was also observed at the multivariate model. However, parents'/caregivers' underestimation of associated with increase in BMI percentile $(1 \cdot 72,95 \% \mathrm{CI}$ 
Table 3 Effect of parental characteristics and perinatal factors on the change in the age- and sex-specific BMI percentile over the 1-year period of the ToyBox intervention - multivariate modelling*

\begin{tabular}{|c|c|c|c|c|c|c|}
\hline \multirow[b]{2}{*}{ Factor } & \multicolumn{2}{|c|}{ Total sample } & \multicolumn{2}{|c|}{ Boys } & \multicolumn{2}{|c|}{ Girls } \\
\hline & Estimate & $95 \% \mathrm{Cl}$ & Estimate & $95 \% \mathrm{Cl}$ & Estimate & $95 \% \mathrm{Cl}$ \\
\hline Baseline BMI percentile & -0.11 & $-0.13,-0.10$ & -0.12 & $-0.14,-0.09$ & -0.11 & $-0.13,-0.09$ \\
\hline \multicolumn{7}{|l|}{ Treatment arm } \\
\hline Control & Reference & & Reference & & Reference & \\
\hline Intervention & 0.34 & $-0.58,1.26$ & 0.50 & $-0.76,1.77$ & 0.10 & $-1 \cdot 01,1 \cdot 22$ \\
\hline \multicolumn{7}{|c|}{ Parents'/guardian' perception of child's weight status } \\
\hline Correct estimation & Reference & & Reference & & & \\
\hline Underestimation & $1 \cdot 72$ & $0.22,3.21$ & 2.22 & $0.15,4.28$ & NA & \\
\hline \multicolumn{7}{|l|}{ Parental weight status } \\
\hline Both underweight/normal & Reference & & Reference & & Reference & \\
\hline One parent overweight/obese & 2.34 & $1.50,3 \cdot 18$ & 2.54 & $1.31,3.76$ & $2 \cdot 18$ & $1.03,3.34$ \\
\hline Both parents overweight/obese & $2 \cdot 87$ & $1 \cdot 61,4.13$ & $2 \cdot 68$ & $0.83,4.53$ & $3 \cdot 27$ & $1.55,4.99$ \\
\hline \multicolumn{7}{|l|}{ Maternal pre-pregnancy weight status } \\
\hline Normal/underweight & Reference & & Reference & & Reference & \\
\hline Overweight/obese & 2.03 & $0.89,3.17$ & 2.05 & $0.37,3.73$ & 1.97 & $0.42,3.52$ \\
\hline \multicolumn{7}{|l|}{ Birth weight } \\
\hline $\begin{array}{l}\text { AGA/LGA } \\
S G A\end{array}$ & & & & & $\begin{array}{c}\text { Reference } \\
-2.75\end{array}$ & $-4.49-1.02$ \\
\hline SGA & NA & & NA & & $-2 \cdot 75$ & $-4.49,-1.02$ \\
\hline
\end{tabular}

AGA, normal for gestational age; LGA, large for gestational age; SGA, small for gestational age.

*Estimates from linear mixed effect models with random intercept for country, socio-economic status and school.

a decrease in their BMI percentile $(-2 \cdot 75 ; 95 \%$ CI -4.49 , -1.02) compared with their appropriate for gestational age and large for gestational age counterparts. There were no significant interaction effects seen between the intervention and any other factors.

\section{Discussion}

The primary aim of the present study was to investigate whether the ToyBox-intervention affected the change in BMI percentile of preschool children from six European countries, over the course of 1 year. However, the intervention was not found to have any significant effect on the change observed in children's BMI percentile. Although some of the targeted EBRB, such as prepacked fruit juice consumption $^{(31)}$ and computer/video games use ${ }^{(32)}$, decreased, this favourable impact of the intervention was not followed by a relevant favourable effect on children's BMI percentile values. The non-significant findings observed in the present study regarding the effect of the intervention on children's weight trajectory could partly be attributed to the fact that the intervention could not modify all targeted EBRB, such as children's physical activity levels ${ }^{(37)}$ and TV viewing ${ }^{(32)}$. Parental lack of concern may play a part, as $<10 \%$ of children were overweight/ obese at baseline and parents of younger children may believe that excessive adipose tissue is typical during development and that overweight/obese children will outgrow their excess body weight ${ }^{(38)}$.

The accuracy of parental perceptions of their child's weight status could be an important determinant that might have influenced children's BMI percentile, since parental misperception of their child's body weight can affect relevant $\mathrm{EBRB}^{(7)}$. The percentage of parents who underestimated the weight status of their overweight/obese children was found to be quite high in the present study (70.6\%) and is comparable to relevant percentages reported by a meta-analysis $^{(6)}$ of seventy-eight studies (i.e. $67.5 \%$; $95 \%$ CI $62.9,71.7 \%$, unadjusted). The majority of studies that examine parental misperception of their child's weight status ${ }^{(6,7)}$ and factors associated with misperceptions ${ }^{(39)}$ have been cross-sectional, while the evidence coming from prospective studies is limited. Regarding the role of parental misperceptions on childhood obesity, it has been hypothesised that correct perception of child's weight status would help parents realise that they need to promote and model healthier behaviours, as a means to improve the child's weight trajectory ${ }^{(6)}$. However, this is not confirmed by the results from other studies. An Australian longitudinal study ${ }^{(40)}$ in 3557 children and their parents, reported that children whose parents perceived their 4-5-year-old children as 'overweight', gained more weight across the 8-year follow-up than those perceived as 'normal weight', while a similar, though non-significant, result was also reported by another study ${ }^{(41)}$. A Dutch study ${ }^{(42)}$ looked at accurate parental perception of child's body weight at the age of 5 years and subsequent changes in the child's BMI $z$-score over time. While $85 \%$ of the parents underestimated their overweight child's weight status, accurate weight status perception was associated with a greater increase in children's BMI from 5 until 9 years of age, compared with the underestimation of the child's weight status. In this regard, and despite the fact that the present study showed that underestimation of weight status is associated with an increase of children's BMI percentile over this important preschool year, previous evidence suggests that parental awareness of the child's overweight status is not necessarily protective 
against subsequent increases in body weight. On the contrary, a recent systematic literature review suggested that interventions aiming to correct parental misperceptions of children's weight status, especially in younger age groups, will most likely not be effective ${ }^{(43)}$.

Although the findings regarding the role of parental perceptions on their child's weight trajectory are inconsistent, the evidence on the role of parental weight status has been reported to be much stronger. Both paternal and maternal overweight and obesity have been shown to increase the risk of preschool obesity in several countries worldwide, including Greece ${ }^{(9)}$, the $\mathrm{UK}^{(11)}$ and $\operatorname{Iran}^{(10)}$. Parental overweight and obesity is also an important risk factor for an unfavourable growth trajectory during childhood. In this context, a large study conducted in the $\mathrm{UK}^{(12)}$ showed that the increase in the BMI of preschool children between the age of 3 and 5 years was strongly associated with overweight status of either parents. Besides inheritance of genes that confer susceptibility to obesity, parental overweight is also a proxy for shaping the postnatal eating and physical activity environment of children. In most cases, overweight parents create and sustain an 'obesogenic' environment (i.e. high-energetic diets and physical inactivity) for themselves and their children ${ }^{(20)}$.

Extensive research that has been conducted during the last decades in the field of the early origins of chronic disease has produced a wide range of evidence and relevant theoretical models that highlight the predisposing role of several perinatal factors on the later manifestation of childhood obesity. These theoretical models suggest that the effect of certain environmental factors during specific, critical periods of early development can lead to permanent physiological and metabolic adaptations that although serve the purpose of improving the chances of fetal and postnatal survival, may become detrimental in the long term and may be expressed at different life stages in the presence of certain environmental influences ${ }^{(44)}$. In this regard, the present study reported mother's pre-pregnancy overweight/obesity status to be one of the strongest perinatal factors that remained positively associated with children's BMI percentile change at the multivariate model. Mother's pre-pregnancy BMI is a well-established risk factor which has been associated with accelerated growth trajectory ${ }^{(45)}$, abdominal adiposity ${ }^{(17)}$ and obesity at preschool years ${ }^{(20)}$. Although gestational weight gain has also been associated with obesity in preschool children ${ }^{(46)}$, the univariate association observed in the present between gestational weight gain above IOM recommendations and increases in boy's BMI percentile became non-significant after including mother's prepregnancy BMI into the model. This suggests that mother's pre-pregnancy weight status is a stronger perinatal determinant of children's weight trajectory at preschool years, a finding that is also supported by a recent meta-analysis which combines a wide range of data from various countries worldwide ${ }^{(23)}$.
The results of the present study derived from the multivariate model also revealed a significant association between size at birth and BMI change at preschool years in girls. More specifically, girls born small for gestational age decreased their BMI percentile compared with girls who were born large for gestational age or appropriate for gestational age. The associations observed between size at birth and adiposity at later life are complex, with the majority of studies however reporting a 'J'-shaped positive association between birth weight and children's weight status $^{(14)}$. Regardless of the exact associations, as size at birth is the product of the nutritional and hormonal milieu in which the fetus develops ${ }^{(44)}$, better control of the trajectory of intrauterine growth via the optimisation of maternal nutrition and health status during pregnancy is fundamental.

The present study should be interpreted under the light of its limitations and strengths. The main limitation was that the ToyBox-sample is not fully representative of the source population of preschool children in Europe, due to the sampling of study participants from specific regions in each country. Further limitations include the possibility of recall bias in the assessment of perinatal factors and the reliance on self-reported parental height and body weight data, although this is mitigated by the use of questionnaires with moderate-to-excellent reliability. Regarding strengths, the main study outcome, that is, the change in the child's BMI percentile, was based on reliable measurements conducted by rigorously trained researchers who achieved excellent intra- and inter-observer reliability ${ }^{(47)}$. Strengths also include the large sample of preschooler children and the cluster-randomised pre- and post-test design including an intervention and control group, as well as the simultaneously consideration of several factors that were previously identified as potential correlates of preschool children's BMI.

\section{Conclusion}

The present study showed that parental overweight status and parental underestimation of their child's body weight constitute unfavourable conditions for the BMI trajectory in preschool years. While the ToyBox intervention did not affect BMI percentile change in preschool children, the significant favourable findings observed by ToyBoxintervention implementation, as well as its low resource requirements and use of available personnel and infrastructure, indicate its scalability potential. It is currently expanding in more regions and countries globally, that is, in Argentina, Belgium, Boston, Bulgaria, Ecuador, Estonia, Germany, Greece, Italy, Malaysia, Malta, New Zealand, Nicaragua, Poland, South Africa, Scotland, Spain and $\mathrm{UK}^{(48,49)}$. Although not fully supported by the available literature, increasing parental awareness on the health risks associated with overweight might help increase readiness for change, thus positively modifying EBRB that can support a healthier weight status for the entire 
family. Furthermore, considering the significant positive associations observed between mother's pre-pregnancy weight status and children's BMI percentile change at preschool age, any initiatives that can support women to enter pregnancy with a normal body weight but also adopt and maintain a healthy lifestyle for them and their children after birth, could be proved to be effective early preventive measures in tackling childhood obesity.

\section{Acknowledgements}

Acknowledgements: ToyBox-study group: Coordinator: Yannis Manios; Project Manager: Odysseas Androutsos, Steering Committee: Yannis Manios, Berthold Koletzko, Ilse De Bourdeaudhuij, Mai Chin A Paw, Luis Moreno, Carolyn Summerbell, Tim Lobstein, Lieven Annemans, Goof Buijs; External Advisors: John Reilly, Boyd Swinburn, Dianne Ward; Harokopio University (Greece): Yannis Manios, Odysseas Androutsos, Eva Grammatikaki, Christina Katsarou, Eftychia Apostolidou, Anastasia Livaniou, Katerina Lymperopoulou, Eirini Efstathopoulou, ChristinaPaulina Lambrinou, Angeliki Giannopoulou, Evita Siatitsa, Efstathoula Argiri, Konstantina Maragkopoulou, Athanasios Douligeris; Ludwig Maximilians Universitaet Muenchen (Germany): Berthold Koletzko, Kristin Duvinage, Sabine Ibrügger, Angelika Strauß, Birgit Herbert, Julia Birnbaum, Annette Payr, Christine Geyer; Ghent University (Belgium): Department of Movement and Sports Sciences: Ilse De Bourdeaudhuij, Greet Cardon, Marieke De Craemer, Ellen De Decker, Department of Public Health: Lieven Annemans, Stefaan De Henauw, Lea Maes, Carine Vereecken, Jo Van Assche, Lore Pil; VU University Medical Center EMGO Institute for Health and Care Research (the Netherlands): EMGO Institute for Health and Care Research: Mai Chin A Paw, Saskia te Velde; University of Zaragoza (Spain): Luis Moreno, Theodora Mouratidou, Juan Fernandez, Maribel Mesana, Pilar De Miguel-Etayo, Esther M. González-Gil, Luis Gracia-Marco, Beatriz Oves; Oslo and Akershus University College of Applied Sciences (Norway): Agneta Yngve, Susanna Kugelberg, Christel Lynch, Annhild Mosdøl, Bente B Nilsen; University of Durham (UK): Carolyn Summerbell, Helen Moore, Wayne Douthwaite, Catherine Nixon; State Institute of Early Childhood Research (Germany): Susanne Kreichauf, Andreas Wildgruber; Children's Memorial Health Institute (Poland): Piotr Socha, Zbigniew Kulaga, Kamila Zych, Magdalena Góźdź, Beata Gurzkowska, Katarzyna Szott; Medical University of Varna (Bulgaria): Violeta Iotova, Mina Lateva, Natalya Usheva, Sonya Galcheva, Vanya Marinova, Zhaneta Radkova, Nevyana Feschieva; International Association for the Study of Obesity (UK): Tim Lobstein, Andrea Aikenhead; CBO B.V. (the Netherlands): Goof Buijs, Annemiek Dorgelo, Aviva Nethe, Jan Jansen; AOK- Verlag (Germany): Otto Gmeiner, Jutta Retterath, Julia Wildeis, Axel Günthersberger; Roehampton University (UK): Leigh Gibson; University of Luxembourg (Luxembourg): Claus Voegele. Financial support: The ToyBox-study was funded by the Seventh Framework Programme (CORDIS FP7) of the European Commission under grant agreement $n^{\circ} 245200$. The content of this article reflects only the authors' views, and the European Community is not liable for any use that may be made of the information contained therein. Conflicts of interest: The authors declare no conflicts of interest. Authorship: Y.M., E.K., C.M., L.A.M., V.I., A.Ś.L., B.K., G.C. and O.A. designed, obtained funding and conducted the ToyBox Study. K.A.L. and G.M. led the analysis of data. All authors contributed to interpreting of the data, drafting the manuscript, to the intellectual content and revising of the final draft of the manuscript. The final version of the manuscript was approved by all the authors. Ethics of buman subject participation: The ToyBox-study (www. toybox-study.eu) adhered to the Declaration of Helsinki and the conventions of the Council of Europe on human rights and biomedicine. Approval for the study was obtained by the following entities: in Belgium by the Medical Ethics Committee of the Ghent University Hospital (20·March·2012, number 2012/110); in Bulgaria by the Ethics Committee of the Medical University of Varna (21.July·2011, number 15); in Germany by the Ethics Committee of the Ludwig Maximilian University of Munich (11·April-2012/Project number: 400-11); in Greece by the Bioethics Committee of Harokopio University (28.February-12 -2010) and the Greek Ministry of Education (21.December-2011, 135559/Г7); in Poland by the Bioethics Committee of the Children's Memorial Health Institute and the Department of Information and Publicity of the Polish Ministry of Education (number 1/ $\mathrm{KBE} / 2012$ ); and in Spain by the Clinical Research Ethics Committee and the Department of Consumers' Health of the Government of Aragón (31·August-2011, number 12/ 2011). All headmasters/teachers and parents/caregivers provided a signed consent form before being enrolled in the study.

\section{Supplementary material}

For supplementary material accompanying this paper visit https://doi.org/10.1017/S1368980021001518

\section{References}

1. Hruby A \& Hu FB (2015) The epidemiology of obesity: a big picture. Pharmacoeconomics J 33, 673-689.

2. Reilly J \& Kelly J (2011) Long-term impact of overweight and obesity in childhood and adolescence on morbidity and premature mortality in adulthood: systematic review. Int J Obes 35, 891-898.

3. Llewellyn A, Simmonds M, Owen CG et al. (2016) Childhood obesity as a predictor of morbidity in adulthood: a systematic review and meta-analysis. Obes Rev 17, 56-67. 
4. Reilly JJ (2008) Physical activity, sedentary behaviour and energy balance in the preschool child: opportunities for early obesity prevention: Symposium on 'Behavioural nutrition and energy balance in the young'. Proc Nutr Soc 67, 317-325.

5. te Velde SJ, Van Nassau F, Uijtdewilligen L et al. (2012) Energy balance-related behaviours associated with overweight and obesity in preschool children: a systematic review of prospective studies. Obes Rev 13, 56-74.

6. Lundahl A, Kidwell KM \& Nelson TD (2014) Parental underestimates of child weight: a meta-analysis. Pediatrics 133, e689-e703.

7. Duncan DT, Hansen AR, Wang W et al. (2015) Change in misperception of child's body weight among parents of American preschool children. Child Obes 11, 384-393.

8. Campbell KJ, Crawford DA \& Ball K (2006) Family food environment and dietary behaviors likely to promote fatness in 5-6 year-old children. Int J Obes 30, 1272.

9. Manios Y, Costarelli V, Kolotourou M et al. (2007) Prevalence of obesity in preschool Greek children, in relation to parental characteristics and region of residence. BMC Public Health 7, 178.

10. Koomanaee S, Tabrizi M, Naderi N et al. (2016) Parental anthropometric indices and obesity in children. Acta Med Iranica 54, 270-275.

11. Semmler C, Ashcroft J, van Jaarsveld CHM et al. (2009) Development of overweight in children in relation to parental weight and socioeconomic status. Obesity 17, 814-820.

12. Griffiths LJ, Hawkins SS, Cole TJ et al. (2010) Risk factors for rapid weight gain in preschool children: findings from a UK-wide prospective study. Int J Obes 34, 624.

13. Rinaudo PF \& Lamb J (2008) Fetal origins of perinatal morbidity and/or adult disease. Semin Reprod Med 26, 436-445.

14. Woo Baidal JA, Locks LM, Cheng ER et al. (2016) Risk factors for childhood obesity in the first 1000 days: a systematic review. Am J Prev Med 50, 761-779.

15. Dubois L \& Girard M (2006) Early determinants of overweight at 4.5 years in a population-based longitudinal study. Int $J$ Obes 30, 610.

16. Hawkins SS, Cole TJ, Law C et al. (2009) An ecological systems approach to examining risk factors for early childhood overweight: findings from the UK Millennium Cohort Study. J Epidemiol Community Health 63, 147-155.

17. Jacota M, Forhan A, Saldanha-Gomes C et al. (2017) Maternal weight prior and during pregnancy and offspring's BMI and adiposity at 5-6 years in the EDEN mother-child cohort. Pediatr Obes 12, 320-329.

18. Janjua NZ, Mahmood B, Islam MA et al. (2012) Maternal and early childhood risk factors for overweight and obesity among low-income predominantly black children at age five years: a prospective cohort study. J Obes. doi: 10.1155/2012/ 457173J.

19. Salsberry PJ \& Reagan PB (2005) Dynamics of early childhood overweight. Pediatrics 116, 1329-1338.

20. Whitaker RC (2004) Predicting preschooler obesity at birth: the role of maternal obesity in early pregnancy. Pediatrics 114, e29-e36.

21. Yu Z, Han S, Zhu J et al. (2013) Pre-pregnancy body mass index in relation to infant birth weight, offspring overweight/obesity: a systematic review, meta-analysis. PLoS One 8, e61627.

22. Tie H-T, Xia Y-Y, Zeng Y-S et al. (2014) Risk of childhood overweight or obesity associated with excessive weight gain during pregnancy: a meta-analysis. Arch Gynecol Obstet 289, 247-257.

23. Voerman E, Santos S, Golab BP et al. (2019) Maternal body mass index, gestational weight gain, and the risk of overweight and obesity across childhood: an individual participant data meta-analysis. PLoS Med 16, e1002744.
24. Dürmuş B, Kruithof CJ, Gillman MH et al. (2011) Parental smoking during pregnancy, early growth, and risk of obesity in preschool children: the Generation R Study. Am J Clin Nutr 94, 164-171.

25. Oken E, Levitan EB \& Gillman MW (2008) Maternal smoking during pregnancy and child overweight: systematic review and meta-analysis. Int J Obes 32, 201-210.

26. Gaillard R, Rurangirwa AA, Williams MA et al. (2014) Maternal parity, fetal and childhood growth, and cardiometabolic risk factors. Hypertension 64, 266-274.

27. Kitsantas P \& Gaffney KF (2010) Risk profiles for overweight/obesity among preschoolers. Early Hum Dev 86, 563-568.

28. Baran J, Weres A, Czenczek-Lewandowska E et al. (2019) Early eating patterns and overweight and obesity in a sample of preschool children in South-East Poland. Int J Environ Res Public Health 16, 3064.

29. Hörnell A, Lagström H, Lande B et al. (2013) Breastfeeding, introduction of other foods and effects on health: a systematic literature review for the 5 th Nordic Nutrition Recommendations. Food Nutr Res 57, 20823.

30. Nascimento VG, da Silva JPC, Ferreira PC et al. (2016) Maternal breastfeeding, early introduction of non-breast milk, and excess weight in preschoolers. Rev Paul Pediatr 34, 454-459.

31. Pinket AS, Van Lippevelde W, De Bourdeaudhuij I et al. (2016) Effect and process evaluation of a cluster randomized control trial on water intake and beverage consumption in preschoolers from six European Countries: the ToyBoxStudy. PLoS One 11, e0152928.

32. Latomme J, Cardon G, De Bourdeaudhuij I et al. (2017) Effect and process evaluation of a kindergarten-based, familyinvolved intervention with a randomized cluster design on sedentary behaviour in 4- to 6- year old European preschool children: the ToyBox-study. PLoS One 12, e0172730.

33. Manios Y, Androutsos O, Katsarou C et al. (2014) Designing and implementing a kindergarten-based, family-involved intervention to prevent obesity in early childhood: the ToyBox-study. Obes Rev 3, 5-13.

34. De Miguel-Etayo P, Mesana MI, Cardon G et al. (2014) Reliability of anthropometric measurements in European preschool children: the ToyBox-study. Obes Rev 15, 67-73.

35. Mouratidou T, Miguel ML, Androutsos O et al. (2014) Tools, harmonization and standardization procedures of the impact and outcome evaluation indices obtained during a kindergarten-based, family-involved intervention to prevent obesity in early childhood: the ToyBox-study. Obes Rev 15, 53-60.

36. Institute of Medicine. (2009) Weight Gain During Pregnancy: reexamining the Guidelines. Washington, DC: The National Academy Press.

37. De Craemer M, Verloigne M, De Bourdeaudhuij I et al. (2017) Effect and process evaluation of a kindergartenbased, family-involved cluster randomised controlled trial in six European countries on four- to six-year-old children's steps per day: the ToyBox-study. Int J Behav Nutr Phys Act 14, 116.

38. Chang LY, Mendelsohn AL, Fierman AH et al. (2017) Perception of Child Weight and Feeding Styles in Parents of Chinese-American Preschoolers. I Immigr Minor Health 19, 302-308.

39. Maynard LM, Galuska DA, Blanck HM et al. (2003) Maternal perceptions of weight status of children. Pediatrics 111, 1226-1231.

40. Robinson E \& Sutin AR (2016) Parental perception of weight status and weight gain across childhood. Pediatrics 137, e20153957.

41. Kroke A, Strathmann S \& Günther ALB (2006) Maternal perceptions of her child's body weight in infancy and early 
childhood and their relation to body weight status at age 7 . Eur J Pediatr 165, 875-883.

42. Gerards SMPL, Gubbels JS, Dagnelie PC et al. (2014) Parental perception of child's weight status and subsequent BMIz change: the KOALA birth cohort study. BMC Public Health 14, 291.

43. Blanchet R, Kengneson C-C, Bodnaruc AM et al. (2019) Factors influencing parents' and children's misperception of children's weight status: a systematic review of current research. Curr Obes Rep 8, 373-412.

44. Godfrey KM \& Barker DJ (2000) Fetal nutrition and adult disease. Am J Clin Nutr 71, 1344S-1352S.

45. Giles LC, Whitrow MJ, Davies MJ et al. (2015) Growth trajectories in early childhood, their relationship with antenatal and postnatal factors, and development of obesity by age
9 years: results from an Australian birth cohort study. Int J Obes 39, 1049.

46. Oken E, Taveras EM, Kleinman KP et al. (2007) Gestational weight gain and child adiposity at age 3 years. Am J Obstet Gynecol 196, 322.e1-322.e8.

47. De Miguel-Etayo P, Mesana MI, Cardon G et al. (2014) Reliability of anthropometric measurements in European preschool children: the ToyBox-study. Obes Rev 3, 67-73.

48. Malden S, Reilly JJ, Hughes A et al. (2020) Assessing the acceptability of an adapted preschool obesity prevention programme: toyBox-Scotland. Child: Care Health Dev $\mathbf{4 6}$, 213-222.

49. Reeves S, Poh BK, Cheah WL et al. (2020) ToyBox Study Malaysia: a feasibility study to improve healthy energy balance, obesity-related behaviour. Proc Nutr Soc 79, E313. 